\title{
Residual Energy and Distance based Energy- Efficient Communication Protocol for Wireless Sensor Network
}

\author{
Atul Pratap Singh \\ M.Tech Scholars, Galgotias \\ University, Greater Noida
}

\author{
Nishu Sharma \\ M.Tech Scholars, Galgotias \\ University, Greater Noida
}

\author{
Nihar Ranjan Roy \\ Assistant Professor, Galgotias \\ University, Greater Noida
}

\begin{abstract}
Wireless sensor network has gained increasing attention for the research community and users in the last years. In a wireless sensor network, sensor nodes have generally limited battery power so the critical aspect is to save the energy of nodes. Routing protocols plays the important role in energy consumption of nodes. In this paper we proposed energy efficient communication protocol based on energy and distance based cluster head selection. In this paper consider the remaining energy of nodes for cluster head selection when nodes are alive far from base station but clustering is not working well for alive nodes which are near to the base station then all nodes select as cluster head and communicate with base station directly. Simulation result with MATLAB show that this approach improves system lifetime 20 to $30 \%$ more than LEACH and save residual energy by an order of magnitude compared to normal approach when energy is limited.
\end{abstract}

Keywords - Clustering methods, energy efficiency, routing protocol, wireless sensor networks.

\section{INTRODUCTION}

In a wireless sensor network, sensor nodes collect the data of various parameters and send to the base station by wireless medium [1]. Because of the latest advances in MEMS-based sensor technology, limited-power electronics device, and low-power RF design have provided the development of inexpensive and low-power wireless micro sensors, but the cost and their size enable applications to network hundreds or thousands of these micro sensors [2] in order to achieve fault- tolerant and high quality sensing networks. Sensor collects the data for a specific parameter like Pressure temperature, humidity, movement, etc. then sensor sends all data to the base station. Are processed at the base station and then the expected amount of the parameter will be estimated. On these networks, the position of sensor nodes need not be manually engineered or pre-determined, which Allows random deployment in inaccessible terrains or disaster relief operations.

\subsection{Design goals for wireless micro sensor}

1.1.1. Network protocols: - For design better protocols for wireless sensor networks. It is important to understand the parameters which are important for the sensor network. There are many ways in which Protocols are beneficial to the application. We use the following metrics.

1.1.2. Easy deployment: -In wireless sensor networks may be hundreds or thousands of nodes and may need to be deployed in dangerous environments or remote area. If these nodes are small and cheap enough we can throw hundreds or thousands of sensor nodes from a plane flying over a remote or dangerous area for extract information in ways that would not have been possible otherwise.

1.1.3. Lifetime of the system: -Wireless sensor networks should active as long as possible system lifetime can be Measured using application specific parameters such as the time until the sensor network is providing acceptable quality results or it can be measured using generic parameters such as the time until the nodes die.

1.1.4. Latency: -Data from sensor networks should be receive in timely manner Long delays due to processing or communication may be unacceptable.

1.1.5Quality: -This parameter measures the accuracy with which the result of the sensor network matches what is actually occurring in the environment. Although this is an application specific and data dependent quantity of possible application independent method of determining quality.

\subsection{Challenge: - Meeting the design goals}

To summarize wireless micro sensor network protocol is should be:-

$>$ Self-configuring for ease of deployment of the sensor node.

$>$ Energy efficient and robust to extend system lifetime.

$>\quad$ Latency aware to get the information quickly as possible. 
$>$ Cognizant of the application specific nature of sensor network quality.

In the wireless sensor network sensors are deployed randomly or may be manually in remote areas. Sensor collects the information for a specific parameter like temperature, pressure, humidity, movement, etc. then sensor sends all data to the base station. After receiving the data base station analyze on the data and get some useful information. Sensors are small electronic device so it has limited battery power. It is not possible to replace the battery of sensor node because it deploy far from the base station in a remote area so replacement cost will greater than the cost of sensor nodes. Then we use the sensor node just as use and through. So our main focus decreases the energy consumption of nodes in communication and increase the lifetime of nodes.

If the sensor node sends the data directly to the base station then those nodes are far from base station dying very soon compare to those nodes which are close to the base station. Because energy consumption depends on the distance of nodes $\left(E T x-a m p \propto d^{\wedge} 2\right)[3]$. If the sensor node sends the data through the fix mediator nodes then those nodes will die very soon which are near to the base station. So overcome of these problem clustering is the solution. LEACH (Low Energy Adaptive Clustering Hierarchy) provides the energy efficiency in communication by dynamic clustering.

In this paper our focus on LEACH (Low Energy Adaptive Clustering Hierarchy). In LEACH protocol use the following clustering model: some of the node selects themselves as a cluster head. These cluster head collect the data from other nodes which are near to the cluster head and finally these cluster head sends the data to the base station. Cluster head changed at every round so it provides the balance energy consumption for all nodes and increase the lifetime of the network.

This paper proposes a modification of LEACH's cluster head selection on the bases of remaining energy of nodes and distance from base station [4] to reduce energy consumption. For a micro sensor network we make the following assumptions [5]:

$>$ The base station (BS) is located far from the sensors or may be in the center.

$>$ All nodes are homogeneous and have limited energy.

$>$ All nodes are able to reach BS

$>$ Symmetric propagation channel

$>$ Cluster-heads perform data compression.

Cluster-heads collect $n$ k-bit messages from cluster nodes and compress the data to cnk-bit messages which are sent to the base station, with $c \leq 1$ as the compression coefficient.

The operation of LEACH has lots of rounds, where each round is separated into two phases, first is the set-up phase and second is steady-state phase. In the setup phase the clusters are organized, while in the steady-state phase data is delivered to the base station. During the set-up phase, each node decides whether or not to become a cluster head for the current round. This paper presents an improvement of LEACH's cluster-head selection and the formation of clusters.

\section{RELATED WORK}

For wireless sensor network LEACH (low energy adaptive clustering) is the most well known energy-efficient clustering protocol that uses coordination in the clustering process. In LEACH protocol sensor nodes organize themselves into local cluster and one of them selected as a cluster head. Cluster head collects the data from other nodes of the cluster and sends to the base station. The operation of LEACH is divided into rounds. Each round has two phases [6] first is the set-up phase when the cluster is organized and the second is steady-state when data are transferred from the nodes to the base station. During the set-up phase a sensor node chooses a random number between 0 and 1 . If this random number is less than the threshold T (n) [7], the sensor node becomes a cluster-head of the current round. The threshold is set as follows:

$$
\mathrm{T}(\mathrm{n})=\left\{\begin{array}{l}
\frac{p}{1-p * r * \bmod \frac{1}{p}} \\
0 \quad \text { otherwise }
\end{array} \text { if } n \in G\right.
$$

Where $P$ is the cluster-head probability, $r$ is the number of the current round and $G$ as the set of nodes that have not been cluster-heads in the last $1 / \mathrm{P}$ rounds. This algorithm ensures that every node select as a cluster-head exactly once within $1 / \mathrm{P}$ rounds.

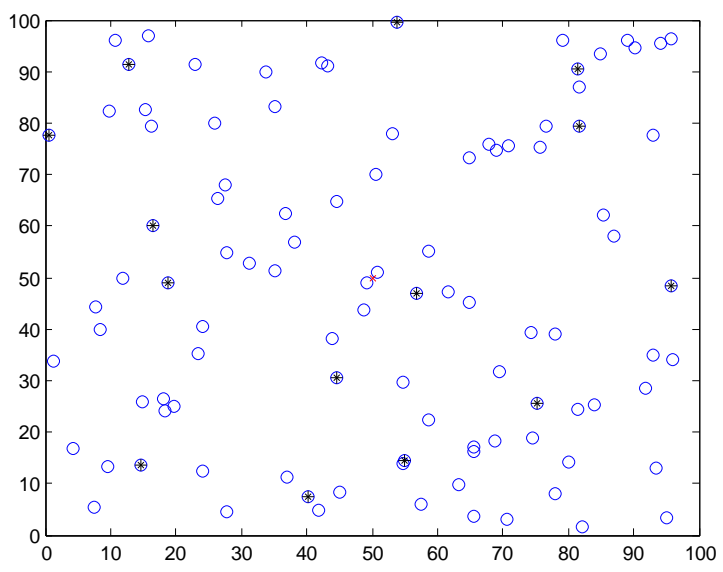

Fig.1. Cluster formation in LEACH

After the cluster head selection, cluster advertises to all nodes that it has selected as cluster head. All nodes create the cluster according to the nearest cluster head. After that during the steady-state cluster head collect the data from other nodes of the cluster (according to TDMA schedule that was created and transmitted to them) and send the aggregate data to the base station. This process ensures that every node becomes a cluster head exactly once within $1 / \mathrm{P}$ 
rounds, that we call this number of rounds as epoch in this paper.

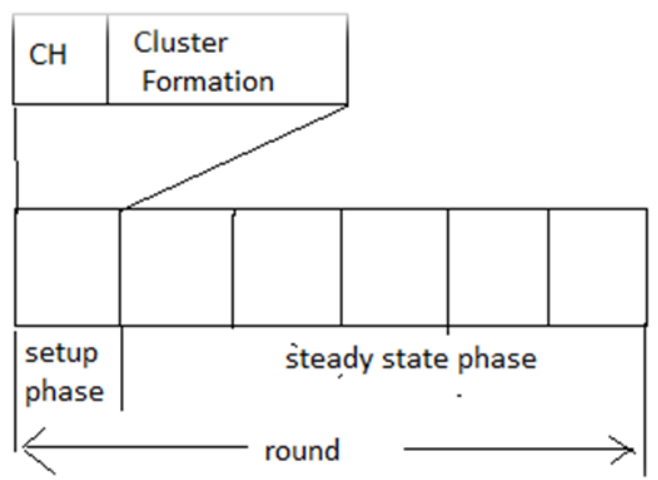

Fig. 2 Time line showing the operation of LEACH

\section{PRELIMINARIES}

\subsection{Radio energy dissipation model}

Modify model use the first order radio model [8] for the radio hardware, energy dissipation which describe that where and how much energy consume in transmitter and receiver part of the sensor nodes to communicate with others node or base station, as shown in Fig.

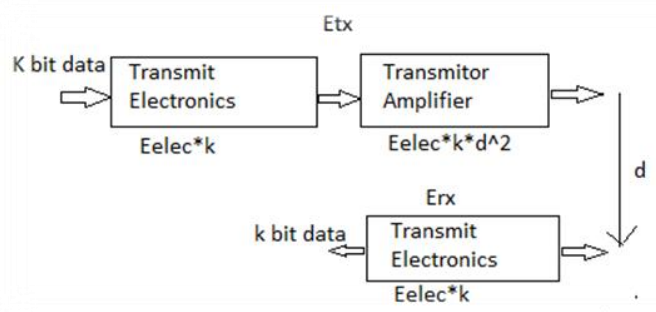

Fig.3. Radio model

The radio characteristics, including energy dissipation in the transmit and receive modes, affect the performance of different protocols. In this paper, the energy model adopted is as follows [9]: the radio dissipates 50 $\mathrm{NJ} / \mathrm{bit}(E \mathrm{elec})$ to run the transmitter or receiver circuitry and $120 \mathrm{pJ} / \mathrm{bit} / \mathrm{m} 2$ ( $E$ amp) for the transmit amplifier. The energy that a node dissipates for the radio transmission $E T x$ $(k, d)$ of a message of $k$ bits over a distance $d$ is due to running both the transmitter circuitry $E$ Tx-Elec $(k)$ and the transmitter amplifier ETX-amp $(k, d)$ and is expressed by the following:
$\mathrm{E}_{\mathrm{Tx}}(\mathrm{k}, \mathrm{d})=\mathrm{E}_{\mathrm{Telec}}(\mathrm{k})+\mathrm{E}_{\mathrm{Tx}-\mathrm{amp}}(\mathrm{k}, \mathrm{d})=\mathrm{E}_{\text {elec }} \cdot \mathrm{k}+\mathrm{E}_{\mathrm{amp}} \cdot \mathrm{k} \cdot \mathrm{d}^{2}$ Where Eelec is the transmitter circuitry dissipation equal to the receiver circuitry dissipation per bit and Eamp is the transmit amplifier dissipation per bit per square meter. Moreover, by using multi-hop routing, $\operatorname{ETx}(k, d)$ is reduced when compared to that in single-hop routing because of distance reduce. Similarly, the energy dissipated by a node for the reception $E \operatorname{Rx}(k)$ of a message of $k$ bits is due to running the receiver circuitry $E \mathrm{Rx}-\mathrm{elec}(k)$ and is expressed by the following equation:

$$
\mathrm{E}_{\mathrm{Rx}}(\mathrm{k})=\mathrm{E}_{\mathrm{Rx} \text {-elec }}(\mathrm{k})=\mathrm{E}_{\text {elec }} \cdot \mathrm{k}
$$

\subsection{Problem formulation}

After the study of LEACH protocol conclusion is that the problem of this protocol is all cluster head sending the data to the base station directly. If all sensor nodes come in the large area, some cluster may be close to the base station and some cluster are far from the base station. This can lead to great differences between the transmission energy dissipation which is used to transmit data to the base station. The radio transmission energy dissipation in two parts first is radio electronics energy and second is power amplifier. Generally power amplifier energy is much larger than the radio electronics energy because power amplifier energy is directly proportional to the distance between nodes.

$$
\left(\mathrm{E}_{\mathrm{Tx}-\mathrm{amp}} \propto \mathrm{d}^{2}\right)
$$

So transmission energy consumption will increase greatly as distance ride. That's mean the cluster head far from the base station use much more energy to send the data compare to those nodes are close.

The second problem of LEACH is that in this protocol node are absolutely chosen accidently or random as a cluster head on the basis of probability and past cluster head selection. Because cluster head consumes a lot of energy, if a node which is chosen has a small energy so this energy will finish quickly and node will be dying very soon. This is the cause for removal of the balance of the network's energy load.

Several clusters-based protocols have been proposed for wireless sensor networks but in none of these protocols (LEACH [10] [11], TEEN [12], APTEEN [13], SOP [14]), consider the residual energy of a node and distance from a base station in the clustering process. Because of this reason, these protocols couldn't distribute energy load in the network in good form. In this paper, by the use of LEACH protocol and considering residual energy and distance parameters in cluster process we proposed new model discuss in below. 


\section{PROPOSED PROTOCOL}

The first approach for solving this problem is that the proposed protocol considers the remaining energy of nodes and the distance from the base station for selection of cluster head. We can find out the distance of every node to the base station by this equation:

$$
d=\sqrt{(S(i) \cdot x d-S(n+1) \cdot x d)^{2}+(S(i) \cdot y d-S(n+1) \cdot y d)^{2}}
$$

Where $\mathrm{d}$ is distance from node to base station $\mathrm{S}(\mathrm{i})$ represent node and $S(n+1)$ is base station.

$100 \%$ node is alive in starting and have same initial energy, hence the node with the less energy than the other nodes have the smallest chance to select as a cluster head for current round. We will consider the remaining energy of sensor nodes in the selection of cluster head.

We will use this threshold equation [15] for selection of cluster head:

temp $=\frac{p}{1-p\left(r * \bmod \frac{1}{p}\right)} * \frac{S(i) \cdot E r}{S(i) \cdot E o}+p *\left(1-\frac{S(i) \cdot E r}{S(i) \cdot E o}\right)$

In this equation $\mathrm{p}$ is the optimal election probability of a node to become the cluster head, or is the current round number, $\mathrm{S}(\mathrm{I})$.Er is remaining energy of nodes and $\mathrm{S}(\mathrm{i})$.Eo is the initial energy of nodes. We use MATLAB for implementation. Our simulations show that such a modification of the cluster-head threshold can increase the lifetime of a LEACH micro sensor network by $15 \%$ for first node dead to the $60 \%$ node dead. Nevertheless, a modification of the threshold-equation by the remaining energy has a crucial disadvantage: After a certain number of rounds or after $60 \%$ dead nodes the network is stuck, although there are still nodes available with enough energy to transmit data to the base station. The reason for this is a cluster-head threshold which is too low, because the remaining nodes have a very low energy level.

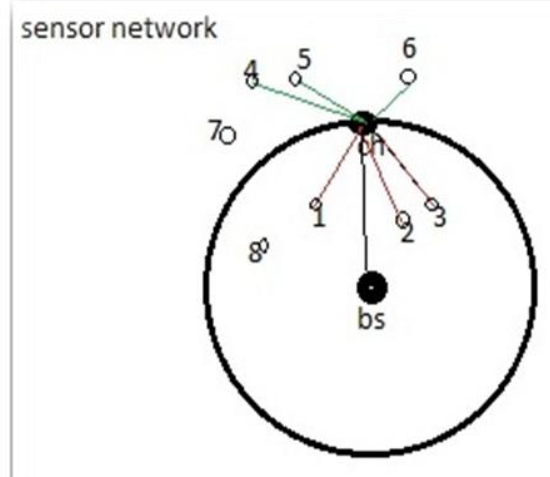

Fig.4. Bad scenario of LEACH
So the second approach to improving the remaining part is that In this figure we want to describe that the inside near region to the base station, clustering not work well because according to this figure node 1,2and 3 communicate to cluster head in opposite direction represented by the red line, this is the energy waste process. These nodes can communicate directly to the base station. . So for the remaining $40 \%$ alive node we will consider in proposed model every node will select as a cluster head [16] at every round then every node to communicate directly to the base station. So after modifying this method implement this protocol again.

We will check the result in two situations.

(1). If the base station is inside the network.

(2). If the base station is outside the network

So first initialize the parameter [17] of this protocol this parameter value is preferred by the past year research for better performance. So these are given below in the table:

\begin{tabular}{|c|c|c|}
\hline Parameter & $\begin{array}{l}\text { Base station (inside the } \\
\text { network) }\end{array}$ & $\begin{array}{l}\text { Base station (outside } \\
\text { the network) }\end{array}$ \\
\hline $\mathrm{Xm}$ (mtr.) & 100 & 100 \\
\hline Ym (meter) & 100 & 100 \\
\hline BS.x & $0.5^{*} \mathrm{xm}$ & $0.0^{*} \mathrm{xm}$ \\
\hline BS.y & $0.5^{*} \mathrm{ym}$ & $0.0^{*} \mathrm{ym}$ \\
\hline N(no. of nodes) & 100 & 100 \\
\hline $\mathrm{Nr}$ & $50 * \operatorname{sqrt}(2)$ & $50 * \operatorname{sqrt}(2)$ \\
\hline $\begin{array}{l}\text { Eo(initial energy in } \\
\text { jule) }\end{array}$ & 0.5 & 0.5 \\
\hline EDA(jule) & $5 * 0.000000001$ & $5 * 0.000000001$ \\
\hline Eelec(jule) & $70 * 0.000000001$ & $70 * 0.000000001$ \\
\hline Eamp(jule) & $0120 * 0.00000000000$ & $\begin{array}{l}0120 * 0.0000000 \\
00001\end{array}$ \\
\hline Rmax(rounds) & 1500 & 1500 \\
\hline $\mathrm{P}$ (probability) & 0.2 & 0.2 \\
\hline $\mathrm{m}$ & 0.2 & 0.2 \\
\hline $\mathrm{a}$ & 1 & 1 \\
\hline
\end{tabular}

Table1. Parameters

\subsection{If the base station is inside the network.}

Sometime it is possible to the established base station inside the sensor network region so first implement this model of this situation. So set the position of the base station is the center of the region and analysis of these protocols on the bases of three parameters, life time, dead nodes, residual energy. And finally compare the result to the LEACH protocols so after the implementation of both protocols we will get these results: 


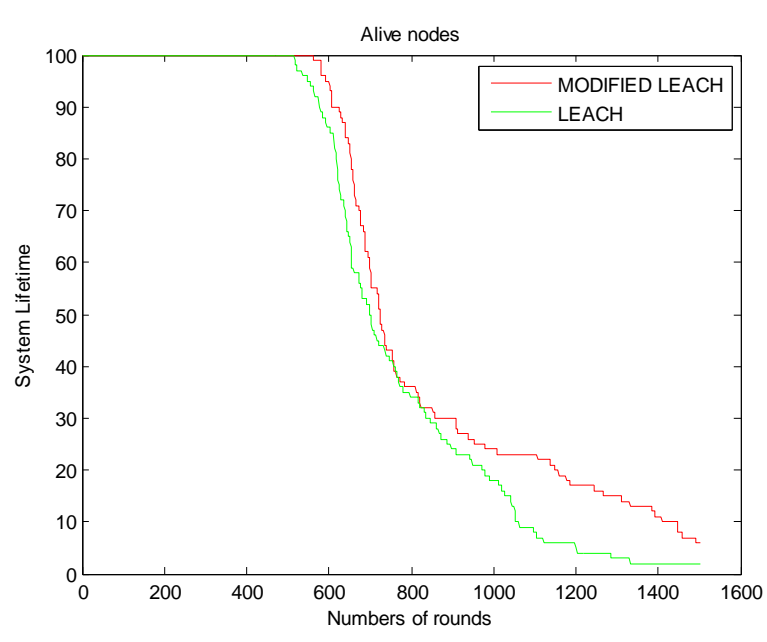

Fig.5 Alive node /round

We can see that lifetime of modified model is $15-20 \%$ more than the LEACH protocol in the first half and 20 to $30 \%$ more in the second half.

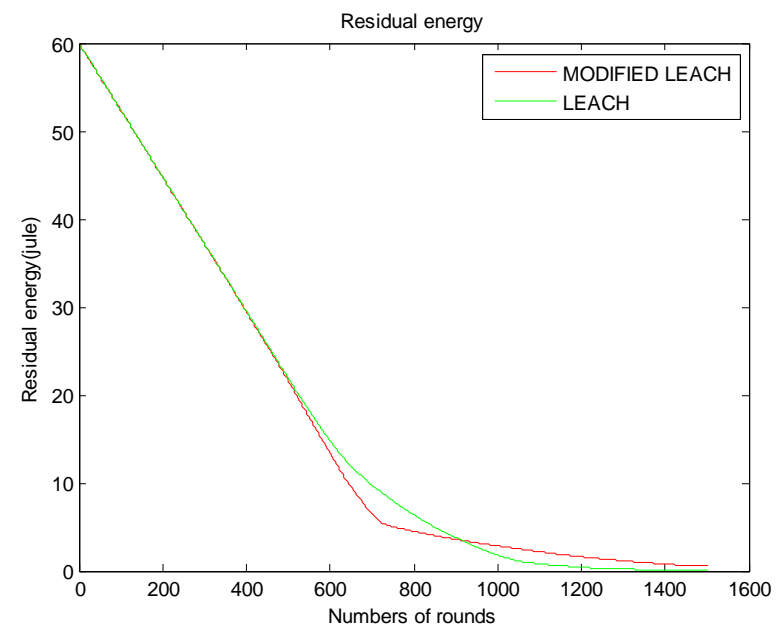

Fig.6. Residual energy

This figure represents the residual energy of the whole network (sum of all nodes residual energy). So we can see that modified model works same as LEACH in starting and better at last.

So conclusion is that modified model is working better than LEACH if the base station is in the center of the network.

\subsection{If the base station is outside of the network}

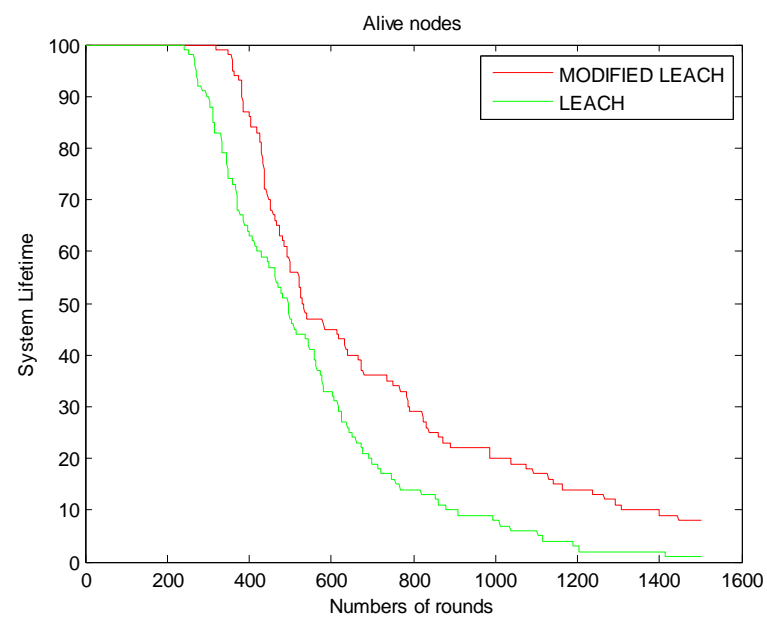

Fig 7. Alive nodes/round

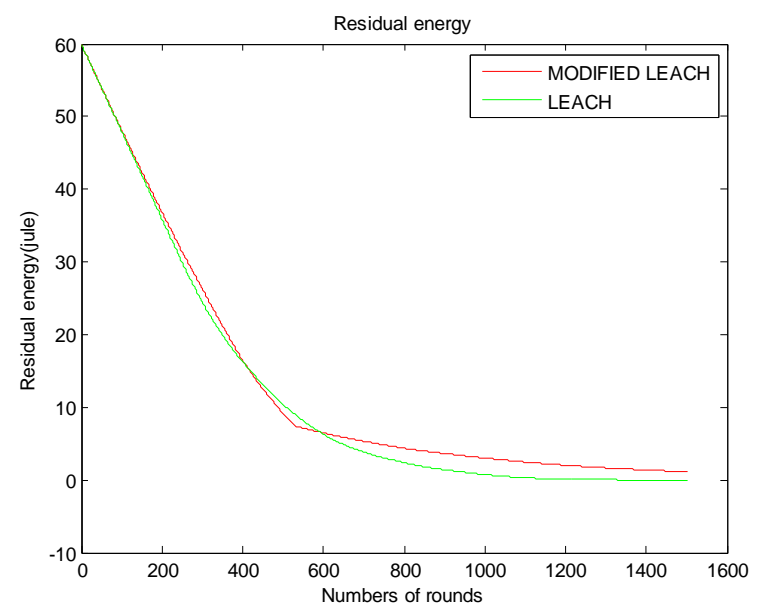

Fig.8. Residual energy

So after the analysis of the implementation results conclusion is that modified model also works well if the base station is outside of the region. It gives 25 to $30 \%$ better results comparable to LEACH protocol that's mean this model work well in both conditions.

This model provides the better load balancing and energy efficiency by the efficient way of cluster head selection on the base of remaining energy of nodes and distant of nodes to the base station. 


\section{CONCLUSION}

This paper present modified LEACH method for WSNs in which consider the energy and distance for cluster head selection and compared it to the LEACH protocols. Conclusion of this paper is that if clustering allowed only far nodes then we get good results of energy effiviency. Result from our simulation show that modified LEACH provides better performance for energy efficiency and network lifetime in both situations when base station in the center and outside of the region of sensor nodes. This modified protocol provides the load balancing of the network. Modified protocol can be classified as a protocol with continuous data transfer just like LEACH in which its general form is intended for static networks. This protocol can be use for multi hope routing. This approach can use in other protocols. In the future work implement this approach in static clustering and analysis the result on the basis of throughput, latency, lifetime, and energy efficiency.

\section{REFERENCES}

[1] I.F. Akyildiz, W. Su*, Y. Sankarasubramaniam, E. Cayirci, 2002, Wireless sensor networks: a survey", Computer Networks 38 393-422

[2]AkyildizI.F.,W.Su,Y.Sankarasubramaniam, E. Cayirci., 2002"Wireless sensor networks: a survey", Journal of Computer Networks, Vol. 38,March 2002, pp. 393422.

[3] Khadivi A. and M. Shiva, 2006 "FTPASC: A Fault Tolerant Power Aware Protocol with Static Clustering for Wireless Sensor Networks", Proc. Of IEEE Int. Conf. on Wireless and Mobile Computing, Networking and Communications, Montreal, Canada, Jun., pp. 397-401.

[4]M. Saeidmanesh, M. Hajimohammadi, and A. Movaghar, 2009" Energy and Distance Based Clustering: An Energy Efficient Clustering Method for Wireless Sensor Networks", World Academy of Science, Engineering and Technology .

[5]. M. J. Handy, M. Haase, D. Timmermann," Low Energy Adaptive Clustering Hierarchy withDeterministic Cluster-Head Selection.

[6] M. Malik,Dr. Y. Singh,2013, "Analysis of LEACH Protocol in Wireless Sensor Networks"IJARCSSE ,Volume 3, Issue 2..

[7] D Kumar,Trilok C. Aseri ,R.B. Patel, (2009)” EEHC: Energy efficient heterogeneous clustered scheme for wireless sensor networks", Computer Communications 32 662-667.

[8]. W. R. Heinzelman, A.Sinha,A. Wang and A. P.Chandrakasan, 2000 "Energy Scalabel Algorithms and Protocols for Wireless Micro sensor Networks".

[9] W. R. Heinzelman, A. Chandrakasan, and H. Balakrishnan, 2000, "Energy-Efficient Communication Protocol for Wireless Micro sensor Networks", IEEE, Maui, Hawaii.

[10] Heinzelman W. R., A. P. Chandrakasan and H. Balakrishnan,2002, "Energy-Efficient Communication Protocol for Wireless Micro sensor Networks",Proc. of the 33rd IEEE Int. Conf. on System Sciences, Honolulu, USA, ., pp. 1-10.

[11] Heinzelman W. R., A. P. Chandrakasan and H. Balakrishnan,2002,“An Application-Specific Protocol Architecture for Wireless Micro sensor Networks", IEEE Trans. on Wireless Communications, vol. 1, no. 4, pp. 660-670.

[12] Manjeshwar A. and D. P. Agarwal,2001 "TEEN: A Routing Protocol forEnhanced Efficiency in Wireless Sensor Networks", Proc. of the IEEEIPDPS, San Francisco, USA, pp 23-26.

[13] Manjeshwar A. and D. P. Agarwal, 2002"APTEEN: A Hybrid Protocol forEfficient Routing and Comprehensive Information Retrieval in Wireless Sensor Networks," Proc. of the IEEE IPDPS, Fort Lauderdale, USA, pp. 195-202.

[14] Subramanian L. and R. H. Katz,2000 "An Architecture for Building Self Configurable Systems", Proc. of IEEE/ACM Workshop on Mobile Ad Hoc Networking and Computing, Boston, USA, pp. 63-73.

[15] Xiaoxiang Bian, Xingcheng Liu, Haengrae Cho," Study on a Cluster-Chain Routing Protocol in Wireless Sensor Networks" IEEE, 2008.

[16] Xuxun Liu," A Survey on Clustering Routing Protocols in Wireless Sensor Networks" Sensors 2012.

[17] Calhoun B. H., D.C. Daly, N. Verma, D.F. Finchelstein, D.D. Wentzloff, A. Wang, S. Cho and A.P. Chandrakasan, "Design Considerations for UltraLow Energy Wireless Micro sensor Nodes", IEEE Trans. On Computers, vol. 54, no. 6, Jun. 2005, pp. 727-740. 